\title{
Improving the Patient-Physician Dialogue through Health Information Systems (HIS): Misconceptions and Miscommunication
}

\author{
Sharon Kishik \\ Aarhus University \\ sharonkishik@hotmail.com
}

\author{
Sune Dueholm Müller \\ Aarhus University \\ $\underline{\text { sdm@processinnovation.dk }}$
}

\author{
Kathrine Stampe \\ Aarhus University \\ $\underline{\mathrm{ks} @ \text { mgmt.au.dk }}$
}

\begin{abstract}
This paper looks at the use of Health Information Systems (HIS) from a communication perspective. Drawing on Niklas Luhmann's systems theory, we analyze patients' self-observations in relation to their disease, as well as physicians' observations of PRO data collected via a mobile application. Based on the analysis, we argue that patient-physician miscommunication occurs, and that the premises of HIS supported information sharing rest on a too simplistic conceptualization of communication. Conclusively, we discuss the implications for the use of HIS instruments in support of patient-physician information sharing, communication, and understanding.
\end{abstract}

\section{Introduction}

In a 2015 study, the OECD finds that the rising healthcare costs in member countries has reached unsustainable levels [17]. As a result, innovation in healthcare is needed to ensure that the quality of healthcare services is not adversely affected [14]. This innovation may in part be driven by digital technologies [15]. Additionally, national healthcare strategies, such as the one in Denmark, emphasize the importance of patients assuming ownership of their healthcare treatment [22]. Concurrently, the literature on digital health focuses on how digital technologies may improve quality of healthcare by supporting patient-centeredness [10]. However, to ensure that digital technologies support value-creation, relevant stakeholders must understand the interests and needs of both patients and physicians. Thus, in this paper, we aim to contribute to this knowledge by focusing in particular on patient-physician information sharing, which we approach from a communication perspective.

Within research on digital health, the question of how to collect and use Patient-Reported Outcome (PRO) data has attracted much attention [15].
According to the FDA, "A patient reported outcome is a direct response from the patient regarding his/her health condition, without a provider or caregiver interpretation" (https://www.fda.gov/patients/learnabout-fda-patient-engagement). At present, physicians frequently rely on periodic surveys and dialogue with patients in order to collect PRO data. However, data is often unreliable due to recall bias affecting answers when patients are asked to account for their well-being over an extended time period [15]. Part of the solution may lie in Healthcare Information Systems (HIS), specifically digital PRO instruments such as mobile applications. These instruments allow patients to continually report observations of a more subjective nature regarding their health. Consequently, the literature on HIS points to benefits such as more reliable PRO data, increased patient engagement and patient-centeredness, and improved communication between patients and physicians. As a result, it is argued that HIS for PRO data management and use improve patient-physician understanding, which may ultimately lead to better health treatment [19].

The question, to which this paper offers a new perspective, is whether HIS for PRO data management and use indeed enable information sharing that leads to greater patient-physician understanding, and whether it affords patients the opportunity to communicate with physicians on their own terms. Our analysis suggests otherwise, and on that basis, we argue that the complexity of patient-physician information sharing requires a new conceptualization of communication if important obstacles are to be solved.

This leads us to our research question: How is the use of digital PRO instruments challenged by obstacles related to communication? Empirically, we address this question in the context of Danish healthcare. More specifically, this paper concerns the use of HIS in the form of a digital PRO instrument, namely the How-Ryou app, which is currently undergoing clinical testing. Drawing on Niklas Luhmann's systems theory, and predominantly his theory of communication, we analyze patient-physician information sharing through the app. Using a Luhmannian frame implies going 
from an understanding of information as ontologically stable toward an understanding of information as ontologically dependent upon observations. Based on our analysis, we argue that information sharing through digital PRO instruments may not improve patientphysician understanding, because miscommunication occurs.

The paper is structured as follows. Firstly, we account for extant literature on HIS and digital PRO instruments with emphasis on information sharing and communication. Secondly, we clarify our theoretical framework which consists of Luhmann's systems theory. Thirdly, we introduce the How-R-you case and the empirical foundation for our analysis. Subsequent to the analysis, we discuss the analytical findings and our contribution to state-of-the-art knowledge. Furthermore, implications for the use of digital PRO instruments are discussed with focus on their potential for improving patient-physician communication and understanding.

\section{Background literature}

Healthcare Information Systems (HIS) are defined as "hardware, software and telecommunication components that support patients in pursuing individual and contextual healthcare goals which leads to both expected and unexpected outcomes" [21:5]. This definition treats health as a dynamic concept which is understood on an individual and contextual basis. Moreover, it aligns with the change in healthcare toward a more patient-centered approach and assigns active agency to patients. Thus, this definition of HIS is relevant to our patient-centered investigation.

Value creation for patients: HIS have the potential to reduce costs for both patients and healthcare organizations. Studies show a reduction in number of hospitalizations and time spent in ambulatory care, because patients gain access to information in support of self-care [18]. In fact, HIS are said to support selfmanagement of diseases and help improve the lives of patients suffering from chronic conditions [4]. Furthermore, studies report on patients who experience that HIS help them describe their health status in greater detail and more objectively than previously [24]. This is in part because HIS facilitate continuous patient input, which has been shown to counter recall bias [15]. HIS help patients record and remember details regarding their health and is an external memory of patients' medical history [23]. Furthermore, HIS empower patients [5] and give them a better understanding of their health situation [16].

Value creation for physicians: the literature emphasizes that HIS not only enable physicians to gain a better understanding of patients but also increase patient-engagement through information sharing [7]. Thereby, HIS support physicians in individualizing healthcare, and enable patients and physicians to make more informed decisions [16].

Co-created value in support of patient-physician collaboration: HIS help establish a mutual understanding between physicians and patients [7]. HIS ease information sharing and make information available to both patients and physicians across time and space [5]. This information allows patients to selfmanage their medical condition and, concurrently, enables physicians to monitor and track patients in all aspects of their daily lives, which supports medical decision-making [13].

In summary, the literature shows that HIS produce a shared knowledge base, which provides the basis for more informed decision-making and greater understanding. In turn, this leads researchers to conclude that HIS help improve patient-physician communication and interaction [5]. Ultimately, it is argued that HIS help improve overall public health [19].

There are various types of HIS for documenting, storing, sharing, and using health data and information. Among these are digital PRO instruments in the form of mobile applications. Generally speaking, mobile technologies are widely used as they support healthcare in numerous ways. Due to their unique characteristics, like mobility and accessibility, they can be used for, e.g., monitoring vital signs and tracking physiological well-being [15]. Furthermore, because these technologies communicate over the Internet, they can give patients a sense of privacy, because patients avoid the stigma of being seen entering medical clinics [6]. PRO data collected through digital instruments may also be considered more accurate, because human errors in the collection or translation process are eliminated [6]. Thus, digital technologies like mobile applications have the ability to more reliably collect and transmit various types of PRO data [6].

In conclusion, the literature points to mostly positive outcomes of HIS use, and improved patientphysician information sharing, communication, and understanding is frequently mentioned. The negative outcomes pertain mainly to instances where HIS have not supported work processes [8]. Yet, obstacles related to communication have, to our knowledge, been largely overlooked. Thus, in this paper, we make a critical contribution by challenging some of the premises and assumptions in the literature. We look at the use of HIS through the lens of Luhmann's systems theory and identify miscommunication between patients and physicians. Consequently, we contribute by suggesting a different conceptualization of 
communication, which encompasses the complexity of patient-physician information sharing and communication.

\section{Theoretical framework}

In this section, we introduce the concepts of Luhmann's systems theory that frame our paper. We start with a brief presentation of his theory of observation, which leads us to his notion of functional differentiation. Subsequently, we account for Luhmann's theory of communication, which frames our analysis of patient-physician information sharing.

Rather than basing his theory on being, Luhmann gives primacy to the epistemological question of becoming. Luhmann replaces classical dichotomies such as subject-object and perception-reality with a question of observation. An observation is defined "as an indication within a frame of difference" [1:7]. When something is indicated, a distinction is always made to something else (see Figure 1). The indication is defined only in relation to that from which it is distinguished. Any observation is observed from a blind spot. The blind spot is the unity of that distinction, which the observer uses to indicate something in the world. In systems theory, the unity of the distinction is called the form of the observation, and it dictates how the world comes into being through observation. In essence, Luhmann's systems theory is about identifying the blind spots of observations in order to understand how meaning is constructed [1]. It enables us to analyze patient-physician communication and observe the blind spots of patients and physicians to understand how they construct different meanings, which in some cases lead to miscommunication. In section 4.2., we elaborate how the theoretical framework guides our analytical approach.

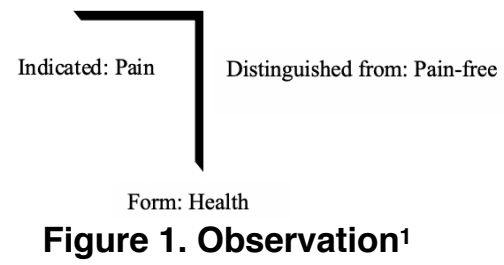

Luhmann is interested in how meaning is constructed in the observations of systems and in particular functional systems [3]. He describes society as being functionally differentiated into communicative, social systems, each serving a particular societal function. For instance, the economic system allocates resources, and the political system produces generally binding decisions [3]. Functional

\footnotetext{
${ }^{1}$ Adapted from Spencer-Brown [20].
}

systems have a number of characteristics. Firstly, they adhere to a specific code. Functional systems each observe through their particular form, called a code. For instance, the health system observes through the binary code health/illness. A code is therefore a binary distinction that splits the world according to a designation value and a reflection value. The designation value is the favored value that participants in communication are motivated to achieve, and the reflection value is the value against which the designation value is defined [1]. Secondly, functional systems are operationally closed around their internal operations and cognitively open to external information. The systems are closed because they operate only according to their own functions and codes. The political system sees the world as a question of power as opposed to powerlessness, whereas the love system understands the world only as a matter of being loved as opposed to not-loved. The point is that the systems represent mutually exclusive forms of communication that each construct the world in specific ways. This means that the codes through which we communicate construct the phenomena that we communicate about [3]. The systems are cognitively open in the sense of taking in external information, but they process this information according to their own logic by observing it through their particular codes. Thus, information is constructed as a product of the system's own observation [11]. Therefore, systems are autopoietic, i.e. self-producing. Functional systems come into being only by distinguishing themselves from their environment. Each system thus creates its own environment as a result of its observations. This means that functional systems are self-producing systems that uphold their autopoiesis by referring to self-produced elements in a self-constructed environment [3].

Using a Luhmannian frame to analyze information sharing enables us to analyze how communication differentiates in completely different and closed forms that construct meaning in fundamentally disparate ways. This allows us to compare how patients and physicians observe, and thus how they assign meaning to information. We do so by analyzing the process and sequence of information sharing in terms of Luhmann's theory of communication.

First and foremost, this entails abandoning any classical, normative notion of communication as a transmission between cognitions. In Luhmannian terms, cognitions are psychic systems that are selfreferential and operationally closed. This means that information cannot be included in the system without it first being transformed into a product of the system's own observation. Consequently, a direct transmission is a theoretical impossibility [9]. Instead, Luhmann 
defines communication as a synthesis of three selections, namely "selection of information, selection of the utterance of this information, and a selective understanding or misunderstanding of this utterance and its information" [12:252]. A selection means that one possibility is selected among numerous others. So, one particular piece of information is selected among different possibilities. This information may be represented in writing as opposed to uttered by speech, and the information may be interpreted and understood in numerous ways. Communication is created only in so far as all three selections are made [12].

By employing Luhmann's theory of communication, we are able to analyze patientphysician information sharing not as a transmission but as a process where participants make selective choices. Thereby, we avoid reducing patient information to something given, i.e. ontologizing information, as well as confusing understanding with reception of message. Accordingly, in this paper, we use the term miscommunication in instances where informationand thus meaning-is constructed in fundamentally different ways on each side of the communication.

\section{The How-R-you case}

The How-R-you case pertains to a Danish research project concerning children and adolescents diagnosed with juvenile arthritis. The study is an ethnographic field study about how technology supports patients in recording relevant information and sharing it with physicians. As part thereof, the study contributes to the development and adoption of the How-R-you app. The app is used by physicians in order to gain a better understanding of the patients through reliable PRO data. Such data are particularly important in treating chronic diseases because physicians need to continuously monitor the patients [15]. The How-Ryou app allows patients to report on their health and well-being on a continuous basis, and it may therefore substitute the use of periodic surveys and solve the aforementioned reliability issue [15].

The app is organized in modules containing health related questions. The modules include "My day", "My night", "My medicine", and "My pain". The modularized questions as well as the home screen (see Figures 2-3) are configurable, allowing patients and physicians to personalize the app according to individual needs. Additionally, the app contains a diary in which patients may write daily notes as free-form text. Furthermore, the app gives patients and physicians an overview of historical data by converting data into graphs and tables. Patients are encouraged to use the app continuously, and the data are examined by the physicians and included in the health assessment of patients during consultations.

The app is available for download here: http://howr-you.online/en/

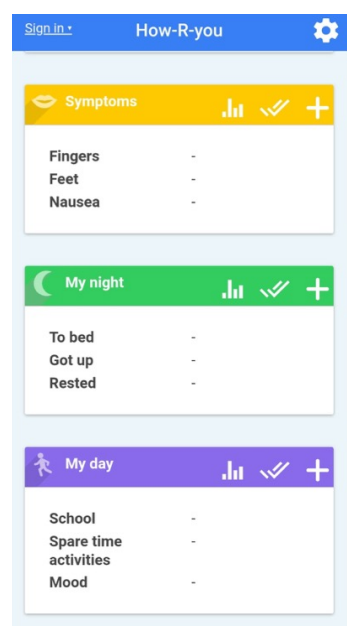

Figure 2. Home screen

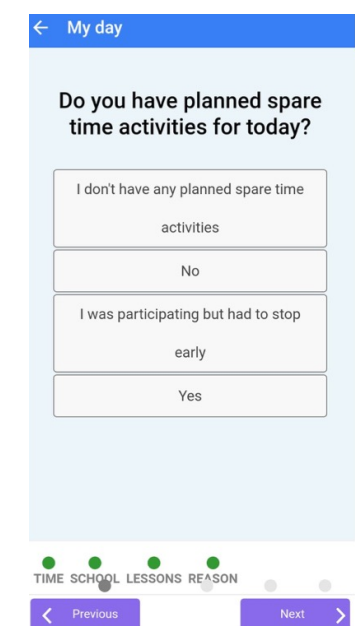

Figure 3. Module

\subsection{Data collection}

Understanding information sharing necessitates knowledge of both what and how patients report through the app as well as the manner in which PRO data are understood and communicated. Therefore, our empirical foundation comprises two sets of data that encompass the different stages of information sharing. The data include 15 interviews with 13 patients and observations of four patient-physician consultations during which the dialogue revolves around the app and the collected PRO data. Patients were selected based on purposeful sampling as part of a larger research project. Participation was voluntary and informed consent was obtained.

Patient interviews: Questions were prepared and grouped by topic in support of the semi-structured interviews. The topics include how the patients are influenced by their disease, how it affects their everyday lives, how they manage pain, and their experiences with medication. Also, the interviews focused extensively on information management through the use of technology. The patients were, for example, asked about their use of technologies in support of information needs in order to ascertain whether and how an app fits with their lifestyles.

The patients are boys and girls in the ages 6-17. The variation among patients is critical to identifying similarities and differences across age groups. Due to the patients' relatively young age, parents were 
occasionally present at the interviews and assisted the patients in answering the questions.

Patient-physician consultations: $35+$ observations were carried out in order to gain an understanding of how these consultations are conducted. Thereby, we were able to compare them to the subsequent "app consultations" and identify differences. At the "app consultations", the patient-physician dialogue revolves around the PRO data, which the physicians observe through the app.

\subsection{Data analysis}

As previously mentioned, Luhmann's systems theory-and our analytical approach-call for observations of blind spots (the forms) of observations [1]. This implies observing observations as observations, i.e. observing in the second-order. The notion of second-order observation is autological, and a second-order observer is not exempt from drawing a distinction [1]. Therefore, we must specify our guiding distinction through which we observe our data, and which-following Luhmann - dictates how the world appears to us. In this paper, we are interested in patients' and physicians' observations, and in how they connect to the communicative codes of functional systems. Therefore, we enter the analysis by making distinctions between what is indicated and unindicated. This means that we observe what is indicated in the observations, what the indications are distinguished from, and thereby we deduce the forms of the observations. All interviews and field notes have been transcribed and coded around themes that are articulated in communication. These include medication, symptoms, pain, and living with the disease. Through second order observations, we analyze how themes are ascribed meaning in communication.

The structure of the analysis follows the three selections in Luhmann's theory of communication. Thus, in the first part of the analysis, we conduct second-order observations of the 15 patient interviews, and we thus examine the patients' selections of information. Thereby, we analyze how patients assign meaning to information that may later comprise the PRO data and be shared through the app. Although this is not an analysis of actual PRO data, the interviews nonetheless revolve around the same topics as the modularized questions. As such, we argue that the same forms of observation are present in the data when app registrations are made. We outline four different observational forms used by patients, each related to a specific functional system. Naturally, the app is preselected as the way in which the information is shared. In other words, this paper is generally concerned with how or whether the selection of the app as a means of sharing information, supports patient-physician communication. We continue by observing the physicians' observations of PRO data during consultations. This relates to the final selection, namely the selection of understanding, i.e. how do physicians observe PRO data through the app, and how do these observations shape communication during consultations. Thereby, we show that the physicians understand patient information in the app through a specific form. This, we argue, results in patientphysician miscommunication.

\section{Analysis}

In this section, we present our analytical findings. Firstly, we show findings from the patient interviews. By including quotations, we illustrate how four distinctive codes are used by patients and their parents. The codes are: power/powerlessness; health/illness; worth it/not worth it; loved/not-loved. Secondly, we present the findings from the consultations, and we show how miscommunication occurs.

\subsection{Patient interviews}

The political system: we begin with the code power/powerlessness, which is associated with the political system. Communication through this code generally concerns how to get something through someone else. In other words, this is communication that focuses on how to exercise power or, conversely, how to resist power [3]. We begin with a quote from the interviews:

"In principle, they cannot control what I will, and what I will not, agree to" - patient

The above quote comes from a patient referring to her physicians. The context is medication, and the patient disagrees with her physicians regarding what to take. The quote is an example of physicians, observed through the code of the political system. Through this code, the physicians are constructed as opponents in a power struggle about who has the last word in decision-making regarding the patient's course of treatment. In a similar vein, when medication becomes the object of the patient's observation through this code, it is constructed as the cause of a power struggle, i.e. as something the patient will not let the physicians force upon her:

"I had a period of time (...) where I lied to my physicians as well as to my mother, where I took the 
needle with me, and then I stuck [ed. and emptied] it in the couch" - patient

Thus, observed through this code, the physicians are observed not as partners or providers of healthcare but as opponents.

In the data, we also found examples of experienced pain, observed through the code of the political system. The following quote comes from a mother who was talking about the importance of physicians understanding how much the disease influences her daughter in her everyday life. Visible pain (patient limping) is constructed as evidence of what she wants the physicians to understand:

"They [ed. the physicians] see it when we enter through the door: well, she has a slight limp (...). Here, it [ed. the influence of the disease] becomes apparent to them" - mother

In a similar vein, the app itself is observed through this code and is, thus, constructed as something the patient can present at the consultation to strengthen her position vis-à-vis the physicians:

“... she [ed. the patient] might realize that it can be a quite good tool when she goes to consultations (...), because then I [ed. the patient] can put it [ed. the app] on the table" - mother

In summary, when the code of the political system is employed in observations related to patients' health and treatment, the different aspects become a matter of power struggles in a Luhmannian sense. This means that the communication revolves around how to get something through the other person.

The economic system: the economic system generally revolves around money which is encapsulated in the code paying/not-paying [3]. In the interviews, the patients do not communicate about money, but the communicative logic of the economic system, namely cost-benefit, is evident. The patients observe through the code worth it/not worth it, and communication becomes about the costs and benefits of treatment and other things related to their health. Observations through the code, worth it/not worth it, are found, e.g., when patients observe their medication and their experienced pain. In the following example, the benefits simply do not outweigh the costs, and thus the patient cannot see the purpose of taking the medication:

"I mean, I was like: well, I feel pain no matter what, so what is the point”-patient
In another example, a mother seems to measure the costs of a specific drug against its benefits. The costs appear greater, and therefore they reject the drug against the physicians' recommendations:

"One might think: well, we want it [ed. the disease] to disappear, but we won't take methotrexate (...). That seems contradictive, one might say, right? However, it's just the costs connected to it, right?" - mother

Moreover, pain itself is observed through this code. In one case, a patient takes a break from her medication and experiences pain. According to her mother, the pain benefits the patient, because she gains an understanding, of why she has to take her medication. Thus, the costs of pain are measured against potential benefits, such as a greater understanding:

"It [ed. the pain] gave her an understanding" mother

When, e.g., medication is observed through this code, it frames the communication in a very specific way. For instance, at the consultations, communication about medication becomes a matter of convincing the patient that the benefits outweigh the costs.

The health system: from the perspective of the health system, the world is observed through the code health/illness, and communication primarily focuses on symptoms and diagnoses [3].

The code of the health system is observed, e.g., when patients are asked how they are influenced by their disease. In these cases, patients often mention impacts such as pain, insomnia, and fatigue. Thus, through this code, these influences are constructed as symptoms of the illness:

"I was really in pain during the night (...) and I couldn't sleep" - patient

"It hurts and it's really hard" - patient

Furthermore, we found observations through this code when patients are asked how an app may help them. Patients say that the app contributes to making them feel less sick:

"Then I am able to think about how I feel at the moment, and I actually feel pretty good" - patient

On the other hand, an app might also make patients feel more ill: 
"I think it will just make it worse (...). Then there are more things, and then... well, then I might be reminded of it more quickly" - patient

Thus, when the app is observed through the code of the health system, it is constructed as something that contributes to either less or more health - to either health or illness.

The love system: The code of the love system is loved/not-loved. Communication through this code is characterized by being personal and intimate. Participants in this communication either fully recognize or reject each other. To fully recognize the other participant means to make him or her one's significant other and to take his or her needs into account. Therefore, communication becomes about anticipating the other's unspoken needs. As a result, love communication depends upon suppositions and anticipatory understanding [2].

In the interviews, patients observe and communicate through the code of the love system, and they show a strong desire not to be reduced to an object of treatment. Patients want to be fully and holistically understood as human beings. In the words of one patient:

"It is just that there is also a person behind the disease" - patient

As a result, when patients mark the consultation as the object of their observation, they describe it as preferably an informal meeting where personal topics are discussed. The consultation becomes a date:

“... it's on a personal level in a way (...). I think it's nice that it's down to earth (...), that it's not just about the arthritis" - patient

When talking about how to make physicians understand the patients as whole people, the app is observed as a tool that patients can use to make the dialogue more personal:

"What we call for [ed. a personal dialogue] may then be unlocked through the app, I believe" - mother

Moreover, the physicians are assigned a radically different role when they are observed through this code. As we know, the codes exclude each other, which means that when a physician is observed as a significant other, he or she cannot simultaneously be observed as, e.g., a provider of healthcare. Thus, in an observation through this code, the treatment is rendered next to irrelevant. In one case, a patient describes her favorite physician who has told her time and time again that he does not know how to treat her disease. However, the physician shows understanding and communicatively recognizes the patient as a whole person. In a Luhmannian sense, the physician shows love:

"You could really see that he was almost sad every time, because he didn't know what to do" - patient

The same physician also shows understanding by anticipating and foreseeing the patient's needs:

"He could almost figure out, before I came in, what the hell I wanted to say" - patient

Given these observations, it seems apparent that the patient-physician relationship is not merely a healthcare provider-receiver relationship. Other expectations are formed in communication, and we shall return to how these expectations may lead to miscommunication. We conclude this section with perhaps the strongest example of communication through the code of the love system. The following quote signals communicatively the ultimate act of love, namely sacrifice:

"He [ed. the physician] was affected, because she cried and stuff. You could see he was almost willing to sacrifice his life just to be able to do something for her" - mother

Our analysis shows, that patients and their parents employ multiple forms when they observe aspects of their health and course of treatment. We proceed by observing the physicians' observations at the consultations.

\subsection{Patient-physician consultations}

In this section, we argue that one specific code dominates the physicians' communication and observations of PRO data. This stands in contrast to the interview findings which show the patients observing and communicating through the app in various codes. Next, we present examples of miscommunication as a result of observations through different codes.

The physicians observe almost exclusively through the code of the health system, namely health/illness. In fact, we found only one example across the four consultations where a physician observed PRO data through another code. This means that when physicians are presented with PRO data through the app, they observe these data through the health/illness code, and they communicate through this code to the patients. Therefore, their communication revolves around 
symptoms and the causes of the symptoms. In one of the consultations, the physician starts by observing the data under the app module "Pain". The physician points out that in the beginning of the recorded timespan, the patient had scored her pain level at " 3 ". However, as the physician notices, the pain level had increased during the timespan. The patient answers by describing the surrounding circumstances during the time when the pain was at its worst. For instance, the patient tells the physician that she had gone with her mother to buy a birthday present for the patient's second cousin. Such information is, however, not considered communicatively relevant to the physician who observes through the code of the health system and is interested in classifying the symptom:

\section{"But did it [ed. her foot] actually lock up?" - physician}

In another example, the physician points out that the patient had reported having slept poorly. Once again, the physician is interested in understanding the symptom, and whether it is connected to the illness:

"Are you in pain or is it just that you are not as comfortable?" - physician

In the following, we demonstrate, how the physicians' observations through this code result in miscommunication.

"Yes, and it's actually not the same day as the one where you were in a lot of pain (...). Nor is it the same day as one of those where you slept poorly" physician

The above statement followed after the physician's observation of the app data, which documented that the patient had reported being in a bad mood. The patient once again responded by explaining the circumstances surrounding the bad mood. The patient had been at work and had experienced low levels of energy. However, the above quote shows that the physician is interested only in how the patient's bad mood is connected to other symptoms and, thus, to the disease. Prior to the above statement, the patient persistently communicates about the circumstances, and she explains that she had been under mental and physical pressure. The physician then realizes that the bad mood is not connected to the disease, and her next comment seems to suggest that it is therefore not communicatively relevant in the consultation. Thus, the physician ends the discussion about mood and assigns the responsibility of improving her mood to the patient herself:
"That's part of what's going to be your challengeto figure out the balancing act in what you are able to do and what you cannot do" - physician

What transpires is a physician who observes through the code of the health system and who communicates about symptoms. In contrast, the patient replies through a different code. The patient tries to make the physician understand what lies behind the symptoms. She communicates through the code of the love system, trying to make the physician recognize her not solely as an object of treatment but as a person.

A similar example was found in a different consultation. The physician recognizes that the patient has reported being in a bad mood. The patient replies:

"But I don't think it was because I was in pain. I don't think so. I think it was related to something at school"-patient

Through the health/illness code, the above reply appears as mere noise, because it does not concern symptoms or diagnoses. Rather, the reply concerns a different aspect of the patient's everyday life. The physician's immediate response illustrates this perfectly. The patient's problems at school are simply not communicatively relevant when seen through the code of the health system. Thus, the physician simply glosses over the patient's remark and returns to symptoms (physical inactivity) that might be connected to the bad mood. Again, the physician communicates through the health/illness code, and the patient is thus rejected by her significant other:

"Yes. Well, that day you weren't as physically active compared to the other days" - physician

Our findings demonstrate that patients and physicians have different expectations. Patients expect physicians to understand them as whole beings. In Luhmannian terms, they expect physicians to love them. However, these expectations are suppressed. Instead, through the code of the health system, the physician constructs the patient as an object of treatment and not a significant other.

\section{Discussion}

Our analytical findings reveal that patients observe aspects of their health and treatment through various codes. In contrast, physicians observe predominantly through the code of the health system. Thus, in some cases, the manners in which information is observed by 
patients, and later understood by physicians, are different. We have presented examples where miscommunication occurs because different codes are used by patients and physicians, leading to different constructions of patient information. This stands in contrast to extant literature in which the assumption is that the use of HIS generally, and digital PRO instruments specifically, leads to improved patientphysician communication $[5,16,19]$ and understanding [7]. Moreover, we suspect that miscommunication happens more often than we can detect using our research methods. Miscommunication is only detectable when patients answer or comment on physicians' communication during the consultations or vice versa. However, when patients record data in the app, these data are observed through various codes, but these observations might not be actualized during the consultations. Physicians who observe through the code of the health system might simply observe these data as noise and not call them to attention during the consultations. As a result, miscommunication goes unnoticed. Moreover, whereas extant research emphasizes that HIS and digital PRO instruments empower patients $[5,7,16]$, our analysis suggests that patients' communicative expectations may be suppressed. Our findings raise the question whether HIS - in this case the How-R-you app_allow patients to communicate on their own terms, or whether they instead allow physicians to determine what is relevant and what is irrelevant information, and thereby to control the consultations. The result of this may be more streamlined consultations, but whether physicians gain a better understanding of the patients is questionable.

This, we argue, presents a challenge, which is not addressed in the literature on HIS supported information sharing. Therefore, we call for more research to investigate both verbal and non-verbal communication during HIS supported patientphysician dialogue. A more systematic understanding of patients' and physicians' communication is needed in order to ascertain patterns, e.g., between patients and forms of communication or discussion topics and forms of communication. By understanding patientphysician communication, we can begin to clarify whether and how HIS and digital PRO instruments support patient-physician communication and understanding.

We argue that a new conceptualization of communication is needed. The conceptualization in extant literature is normative. Communication is seen as a transmission where reception of message equals intended understanding of the message. This leads to the notion that because patient information is made more available, then patient-physician communication is improved $[5,16,19]$. This, we argue, is a problematic premise because information is observed, transmitted, and understood in different ways. By contrast, Luhmann provides a theory of communication that understands communication as a process where participants make contingent, selective choices. Thereby, communication and information sharing may be analyzed not as a transmission but as a process where the manners in which participants observe and communicate construct the phenomena that they communicate about. Thus, by employing Luhmann's theory, researchers may better understand how both patients and physicians construct patient information in communication. Armed with such an understanding, we argue that both the development and the use of HIS and digital PRO instruments for health communication and management should be reevaluated with the aim of better supporting patient-physician communication and understanding. For instance, research should explore novel use of digital PRO instruments. How can digital PRO instruments be set up in a way that allows patients to choose how they want to communicate? Furthermore, how can the use of these instruments during consultations be changed in a way that leads to greater patient empowerment, i.e. patients having more control over the dialogue? Moreover, training physicians in HIS supported communication should be considered. Physicians can be trained to recognize certain key words and phrases that express a certain communicative expectation or need of the patients. For instance, when a patient describes pain in terms of how it influences him or her personally in his or her everyday life, physicians should know that the patient expects to meet understanding and be recognized as a whole person. Such key words and phrases should be identified through comprehensive research of patientphysician communication. In developing specific HIS practices, it is important to realize that codes foster different meanings. Efforts to look beyond specific codes may prevent miscommunication.

\section{Conclusion}

This paper analyzes patient-physician information sharing from a communication perspective. Our analysis shows that communication through different codes leads to instances of miscommunication between patients and physicians. Based on the analysis, we conclude that a different conceptualization of communication is needed. We have proposed Niklas Luhmann's theory that stands in stark contrast to conceptualizations of communication as a transmission. Due to different ways in which patients and physicians assign meaning to information, 
Luhmann's theory better encompasses patientphysician information sharing.

\section{References}

[1] Åkerstrøm Andersen, N., Discursive analytical strategies: understanding Foucault, Koselleck, Laclau, Luhmann, Policy Press, Bristol, 2003.

[2] Åkerstrøm Andersen, N., Flygtige Forhold: om ledelse af medarbejdere mellem paedagogik, kcerlighed og leg, Hans Reitzels Forlag, Copenhagen, 2012.

[3] Åkerstrøm Andersen, N., and J.G. Pors, Niklas Luhmann, Jurist- og økonomiforbundets Forlag, Copenhagen, 2017.

[4] Dadgar, M., and K.D. Joshi, “The Role of Information and Communication Technology in Self-Management of Chronic Diseases: An Empirical Investigation through Value Sensitive Design", Journal of the Association for Information Systems 19(2), 2018, pp. 86-112.

[5] Desmedt, M., S. Pless, E. Dessers, and D. Vandijck, "Integrating and safeguarding care: The potential role of health information technologies", International Journal of Care Coordination 20(3), 2017, pp. 112-118.

[6] Gary, K.A., P. Rallabhandi, Z. Quezado, and K. Cleary, "A Pain Reporting Platform for Adolescents with Sickle-Cell Disease", Proceedings of the 52nd Hawaii International Conference on System Sciences, (2019), 3897-3904.

[7] Huerta, T.R., C. Walker, K.R. Murray, J.L. Hefner, A.S. McAlearney, and S. Moffatt-Bruce, "Patient Safety Errors: Leveraging Health Information Technology to Facilitate Patient Reporting", The Journal for Healthcare Quality (JHQ) 38(1), 2016.

[8] Kim, M.O., E. Coiera, and F. Magrabi, "Problems with health information technology and their effects on care delivery and patient outcomes: a systematic review", Journal of the American Medical Informatics Association 24(2), 2017, pp. 246-250.

[9] Kneer, G., and A. Nassehi, Niklas Luhmanns Theorie sozialer Systeme. Eine Einführung, Wilhelm Fink Verlag, Munich, 1993.

[10] Lee, Y., S.-Y. Shin, J.-Y. Kim, et al., "Evaluation of Mobile Health Applications Developed by a Tertiary Hospital as a Tool for Quality Improvement Breakthrough", Healthc Inform Res 21(4), 2015, pp. 299-306.

[11] Luhmann, N., Soziale Systeme. Grundriss einer allgemeinen Theories, Surhkamp Verlag, Frankfurt am Main, 1984.

[12] Luhmann, N., "What is Communication?", Communication Theory 2(3), 1992, pp. 251-259.
[13] Lupton, D., "The digitally engaged patient: Selfmonitoring and self-care in the digital health era", Social Theory and Health 11(3), 2013, pp. 256-270.

[14] McGowan, J.J., C.M. Cusack, and M. Bloomrosen, “The future of health IT innovation and informatics: a report from AMIA's 2010 policy meeting", Journal of the American Medical Informatics Association: JAMIA 19(3), 2012, pp. 460-467.

[15] Müller, S.D., B. Mahler, and M. Mathiasen, "The Value of Patient-Reported Outcome (PRO) Data in Digital Healthcare: Using the How-R-You App as a PRO instrument", Hawaii International Conference on System Science (HICSS), (2019), 3905-3914.

[16] Nimkar, S., "Promoting individual health using information technology: Trends in the US health system", Health Education Journal 75(6), 2016, pp. 744-752.

[17] OECD, Fiscal Sustainability of Health Systems: Bridging Health and Finance Perspectives, Paris, 2015.

[18] Or, C., R. Valdez, G. Caspar, et al., "Human Factors and Ergonomic Concerns and Future Considerations for Consumer Health Information Technology in Home Nursing Care", Proceedings of the Human Factors and Ergonomics Society Annual Meeting 52(12), (2008), 850-854.

[19] Ranallo, P.A., A.M. Kilbourne, A.S. Whatley, and H.A. Pincus, "Behavioral Health Information Technology: From Chaos To Clarity", Health Affairs 35(6), 2016, pp. 11061113.

[20] Spencer-Brown, G., Laws of Form, Allen and Unwin Ltd, London, 1969.

[21] Stampe, K., and B. Mahler, "Creating health together: how HIS resources can boost co-creation elasticity", Information Systems Research Seminar in Scandinavia (IRIS41), (2018).

[22] SUM, Nationale Mål for Sundhedsvaesenet, 2018.

[23] Waite-Jones, J.M., R. Majeed-Ariss, J. Smith, S.R. Stones, V. Van Rooyen, and V. Swallow, "Young People's, Parents', and Professionals' Views on Required Components of Mobile Apps to Support Self-Management of Juvenile Arthritis: Qualitative Study", JMIR mHealth and uHealth 6(1), 2018, pp. e25.

[24] Young, R., E. Willis, and G. Cameron, “'Willing but Unwilling': Attitudinal barriers to adoption of home-based health information technology among older adults", Health Informatics Journal 20(2), 2014, pp. 127-135. 